Effect of errors in the migration velocity model of PS converted waves on travel time accuracy in prestack Kirchhoff time migration in weak anisotropic media

Hengchang Dai and Xiang-Yang Li

British Geological Survey, Murchison House, West Mains Road, Edinburgh EH9 3LA, UK

Submitted to: Geophysics

Page heading: Error effect in PKTM for anisotropic media

Address for correspondence

Dr. Hengchang Dai

British Geological Survey

Murchison House

West Mains Road

Edinburgh EH9 3LA

UK

Tel: +44 1316500216

Fax: +44 1316671877

Email: hcd@bgs.ac.uk 


\title{
Effect of errors in the migration velocity model of PS converted waves on travel time accuracy in prestack Kirchhoff time migration in weak anisotropic media
}

\author{
Hengchang Dai and Xiang-Yang Li \\ British Geological Survey, West Mains Road, Edinburgh EH9 3LA,
}

Email: hcd@bgs.ac.uk

\begin{abstract}
We investigate the effect of errors in the migration velocity model of PS converted waves on the travel-time calculated in prestack Kirchhoff time migration in weak anisotropic media. The prestack Kirchhoff time migration operator contains four parameters: PS converted wave velocity, vertical velocity ratio, effective velocity ratio, and anisotropic parameter. We derive four error factors corresponding to the four parameters, respectively. Theoretical and numerical analyses of the error factors show that all the error factors are inversely proportional to the velocity as well as to the travel-time. Errors in travel-time for shallow events are usually larger than those for deep events. The error in the PS converted wave velocity causes the largest error in the travel-time, and the error in the vertical velocity ratio causes the smallest error in the travel time. The error in effective velocity ratio has a larger effect on the travel-time than the error in anisotropic parameter when the ratio of horizontal distance to depth is small. However, the error in anisotropic parameter has a large effect on travel-time than the error in effective velocity ratio when the ratio of horizontal distance to depth is lager. The errors in the travel time caused by errors in effective velocity ratio and anisotropy parameter stem mainly from the converted S-wave raypath of the PS converted waves. For time processing, it is possible to estimate PS-wave velocity accurately without accurate information of vertical velocity ratio, effective velocity ratio and anisotropic parameter. This can save processing cost and time. These findings are useful both for our understanding of $P S$-wave behaviour and for PS-wave imaging in anisotropic media.
\end{abstract}


Dai and Li: Error effect in PKTM for anisotropic media

Keywords: anisotropy, converted wave, traveltime, prestack Kirchhoff time migration 


\section{INTRODUCTION}

In isotropic media, the error in the PS wave velocity has a larger effect on the image obtained from prestack Kirchhoff time migration than the error in the P-to-S velocity ratio (Dai and Li 2006). This suggests that for time processing, only the velocity of PS converted waves needs to be accurately estimated and a roughly estimated P-to-S velocity ratio is acceptable for producing the PS converted wave migration image. This phenomenon is useful for processing PS converted wave data because it is difficult and time-consuming to estimate the P-to-S velocity ratio accurately from real data. However, when anisotropy cannot be neglected, there are more parameters in the velocity model of PS converted waves ( $\mathrm{Li}$ and Crampin, 1993; Li and Yuan 2003). We have to investigate the effects of errors in these parameters on the traveltime and imaging of PS converted waves. In this paper, we extend our previous work (Dai and Li 2006) to anisotropic media. Firstly, we analyse the source of errors in the traveltime calculation and derive a relationship between the errors in travel-time and the absolute error of parameters of the migration velocity model. Then we perform a numerical analysis to demonstrate the effects of velocity model error on travel time calculation for prestack Kirchhoff time migration.

\section{EFFECTS OF TRAVEL-TIME ERRORS ON PRESTACK TIME MIGRATION}

The accuracy of PS converted wave travel-times calculated in prestack Kirchhoff time migration plays a crucial role in producing high quality migrated images and is determined by the accuracy of the migration velocity model. For anisotropic media, the migration velocity model consists of PS converted wave velocity $\left(V_{p s}\right)$, vertical velocity ratio $\left(\gamma_{0}\right)$, effective velocity ratio $\left(\gamma_{\text {eff }}\right)$ and the anisotropic parameters $(\chi)$ (Li, et al., 2007; Li and Yuan 2003). 
In prestack Kirchhoff time migration for weak anisotropic media, the travel-time $t_{p s}$ is the summation of the travel-times of the down-going P-wave and up-going S-wave.

$$
t_{p s}=t_{p}+t_{s}
$$

where $t_{p}$ and $t_{s}$ are approximated (see Appendix A) as:

$$
\begin{gathered}
t_{p}=\frac{t_{p s 0}}{1+\gamma_{0}} \sqrt{1+w_{p}-\frac{2 \eta_{\text {eff }}}{1+\left(1+2 \eta_{\text {eff }}\right) w_{p}} w_{p}^{2}}, \\
t_{s}=\frac{\gamma_{0} t_{p s 0}}{1+\gamma_{0}} \sqrt{1+w_{s}-\frac{2 \xi_{\text {eff }}}{1+w_{s}} w_{s}^{2}} .
\end{gathered}
$$

Where $w_{p}=\frac{x_{p}^{2}\left(1+\gamma_{0}\right)\left(1+\gamma_{\text {eff }}\right)}{t_{p s 0}^{2} V_{p s}^{2} \gamma_{\text {eff }}}, w_{s}=\frac{x_{s}^{2}\left(1+\gamma_{0}\right)\left(1+\gamma_{\text {eff }}\right)}{t_{p s 0}^{2} V_{p s}^{2} \gamma_{0}}, \eta_{\text {eff }}=\frac{\chi}{\left(\gamma_{0}-1\right) \gamma_{\text {eff }}^{2}}$ and $\xi_{\text {eff }}=-\frac{\chi}{\gamma_{0}-1}$; $x_{p}$ is the horizontal distance between the source and the scatter point, $x_{S}$ is the horizontal distance between the receiver and the scatter point, and $t_{p s 0}$ is the summation of the vertical travel-time of the P-wave from the source to the scatter point and the vertical travel-time of the S-wave from the scatter point to receiver (Figure 1).

The error in the travel time $\left(t_{p s}\right)$ can be written in terms of the absolute errors in these quantities (see Appendix B):

$$
\Delta t_{p s}=-E_{\gamma 0} \Delta \gamma_{0}-E_{\gamma e f f} \Delta \gamma_{e f f}-E_{v p s} \Delta V_{p s}-E_{\chi} \Delta \chi
$$

where $\Delta V_{p s}, \Delta \gamma_{0}, \Delta \gamma_{e f f}$, and $\Delta \chi$ are absolute errors in $V_{p s}, \gamma_{0}, \gamma_{e f f}$, and $\chi$ respectively, and $E_{v p s}, E_{\gamma 0}, E_{\gamma_{\text {eff }}}$, and $E_{\chi}$ are the corresponding error factors, given by

$$
\begin{gathered}
E_{v p s}=E_{v p s p} t_{p}+E_{v p s s} t_{s}, \\
E_{v p s p}=\frac{1}{V_{p s}} \frac{w_{p}\left[1+2 y_{p}+\left(1+2 \eta_{\text {eff }}\right) w_{p}^{2}\right]}{\left[1+\left(1+2 \eta_{\text {eff }}\right) w_{p}\right]\left[1+2\left(1+\eta_{\text {eff }}\right) w_{p}+w_{p}^{2}\right]}, \\
E_{v p s s}=\frac{1}{V_{p s}} \frac{w_{s}\left[1+2\left(1-2 \xi_{\text {eff }}\right) w_{s}+\left(1-2 \xi_{\text {eff }}\right) w_{s}^{2}\right]}{\left[1+2 w_{s}+\left(1-2 \xi_{\text {eff }}\right) w_{s}^{2}\right]\left[1+w_{s}\right]} ;
\end{gathered}
$$




$$
E_{\gamma 0}=E_{\gamma 0 p} t_{p}-E_{\gamma 0 s} t_{s},
$$

$$
E_{\gamma 0 p}=\frac{1}{2\left(1+\gamma_{0}\right)} \frac{2+\left(5+8 \eta_{\text {eff }}\right) w_{p}+2\left(2+5 \eta_{\text {eff }}+4 \eta_{\text {eff }}^{2}\right) w_{p}{ }^{2}+w_{p}^{3}-\frac{4 \eta_{\text {eff }}\left(1+w_{p}\right) w_{p}^{2}}{\left(\gamma_{0}-1\right)}}{\left[1+2\left(1+\eta_{\text {eff }}\right) w_{p}+w_{p}^{2}\right]\left[1+\left(1+2 \eta_{\text {eff }}\right) w_{p}\right]},
$$

$$
\begin{gathered}
2+5 w_{s}+2 w_{s}^{2}\left(2+2 \xi_{\text {eff }}+\xi_{\text {eff }} \gamma_{0}\right)+w_{s}^{3}\left(1+2 \xi_{\text {eff }} \gamma_{0}+2 \xi_{\text {eff }}\right) \\
E_{\gamma 0 s}=\frac{1}{2\left(1+\gamma_{0}\right) \gamma_{0}} \frac{4 w_{s}^{2}\left(1+w_{s}\right) \xi_{\text {eff }}}{\left(\gamma_{0}-1\right)} \\
{\left[\left(1+2 w_{s}+\left(1-2 \xi_{\text {eff }}\right) w_{s}^{2}\right]\left(1+w_{s}\right)\right.}
\end{gathered} ;
$$

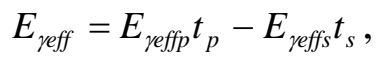

$$
\begin{aligned}
& E_{\text {reffp }}=\frac{1}{2\left(1+\gamma_{\text {eff }}\right) \gamma_{\text {eff }}} \frac{w_{p}\left[1+2\left(1-2 \eta_{\text {eff }}-2 \gamma_{\text {eff }} \eta_{\text {eff }}\right) w_{p}+\left(1-2 \eta_{\text {eff }}-4 \gamma_{\text {eff }} \eta_{\text {eff }}\right) w_{p}^{2}\right]}{\left[1+2\left(1+\eta_{\text {eff }}\right) w_{p}+w_{p}^{2}\right]\left[1+\left(1+2 \eta_{\text {eff }}\right) w_{p}\right]}, \\
& E_{\text {reffs }}=\frac{1}{2\left(1+\gamma_{\text {eff }}\right)} \frac{w_{s}\left[1+2\left(1-2 \xi_{\text {eff }}\right) w_{s}+\left(1-2 \xi_{\text {eff }}\right) w_{s}^{2}\right]}{\left[1+2 w_{s}+\left(1-2 \xi_{\text {eff }}\right) w_{s}^{2}\right]\left(1+w_{s}\right)}
\end{aligned}
$$

$$
\begin{gathered}
E_{\chi}=E_{\chi p} t_{p}-E_{\chi s} t_{s}, \\
E_{\chi p}=\frac{1}{\left(\gamma_{0}-1\right) \gamma_{\text {eff }}^{2}} \frac{w_{p}^{2}\left(1+w_{p}\right)}{\left[1+2\left(1+\eta_{\text {eff }}\right) w_{p}+w_{p}^{2}\right]\left[1+\left(1+2 \eta_{\text {eff }}\right) w_{p}\right]}, \\
E_{\chi s}=\frac{1}{\left(\gamma_{0}-1\right)} \frac{w_{s}^{2}}{\left[1+2 w_{s}+\left(1-2 \xi_{\text {eff }}\right) w_{s}^{2}\right]} .
\end{gathered}
$$

Equation 4 shows that the travel-time error is the summation or substitution of the products of the error factors and the corresponding absolute errors. These error factors illustrate the effects of the relative errors in $V_{p s}, \gamma_{0}, \gamma_{\text {eff }}$, and $\chi$ on the travel-time computation. Their characteristics determine the travel-time dependence. 


\section{NUMERICAL MODEL ANALYSIS}

Generally, each error factor has two terms (Equations 5, 6, 7, and 8); one term is related to the P-wave raypath and the other is related to the S-wave raypath. Note that for weak anisotropic media, the values of these terms are always positive (Equations 5a, 5b, 6a, 6b, 7a, 7b, 8a, and 8b). The error factor of velocity $\left(E_{V_{p s}}\right)$ is the sum of two terms. However, the error factors of velocity ratio ( $E_{\gamma 0}$ and $E_{\gamma_{\text {eff }}}$ ) and anisotropy $\left(E_{\chi}\right)$ are the difference between two terms. This implies that the errors caused by $E_{\gamma 0}, E_{\gamma_{e f f}}$ and $E_{\chi}$ will change sign with common-image-point locations and the error caused by $E_{V_{p s}}$ will not. The sign changes in $E_{\text {reff }}, E_{\gamma_{0}}$ and $E_{\chi}$ mean that when stacking the energy from both negative and positive offset data, $E_{\gamma_{\text {eff }}}, E_{\gamma_{0}}$, and $E_{\chi}$ will cause more smearing than $E_{V_{p s}}$ (Dai and Li 2006). In other words, $E_{\gamma_{\text {eff }}}, E_{\gamma_{0}}$, and $E_{\chi}$ are more likely to affect the focus of the image.

The effects of these error factors on the travel-time calculation can be seen more in numerical evaluation in which the travel-time errors related to $\Delta V_{p s}, \Delta \gamma_{0}, \Delta \gamma_{\text {eff }}$, and $\Delta \chi$ are calculated. The travel-time errors associated with $\Delta V_{p s}, \Delta \gamma_{0}, \Delta \gamma_{\text {eff }}$, and $\Delta \chi$ are calculated for various values of $V_{p s}, \gamma_{0}, \gamma_{\text {eff }}$, and $\chi$ separately. The offset between the source and receiver is set to $1000 \mathrm{~m}$. The locations of common image points are related to the source location and defined as offset/1000m (Figure 1). The source is placed at 0.0 of the location of common image points and the receiver at 1.0 of the location of common-image points. The PS converted wave velocity is set to $1000 \mathrm{~m} / \mathrm{s}$ and $2000 \mathrm{~m} / \mathrm{s}$, and $\gamma_{0}$ and $\gamma_{\text {eff }}$ are set to (2.5, 2.0) and $(4.5,4.0)$. $\chi$ is set to 0.1 and 0.2 for weak anisotropic media. Figure 2 shows the diffraction curves for various values of $V_{p s}, \gamma_{0}, \gamma_{e f f}$, and $\chi$. Each plot in Figure 2 shows five diffraction curves for $1 \mathrm{~s}, 2 \mathrm{~s}, 3 \mathrm{~s}, 4 \mathrm{~s}$, and $5 \mathrm{~s}$ of PS-wave travel-times, respectively. These 
figures show that the diffraction curves obviously vary with velocity, moderately vary with velocity ratio, and vary very small with anisotropy.

Figure 3 shows absolute values of the travel-time error in the PS-wave caused by 10 $\mathrm{m} / \mathrm{s}$ in $V_{p s}(1 \%$ of $1000 \mathrm{~m} / \mathrm{s}$ or $0.5 \%$ of $2000 \mathrm{~m} / \mathrm{s})$ with various $V_{p s}, \gamma_{0}, \gamma_{\text {eff }}, \chi$ and traveltimes. In practice, this error is small. A suitable tool is necessary to pick the velocity in such accuracy. Because $\gamma_{0}, \gamma_{\text {eff }}, \chi$ are true values, the travel-time error is $-E_{V_{p s}} \Delta V_{p s}$. Figure 3 shows travel-time errors caused by errors in velocity are of the same sign, and are inversely proportional to the travel-time itself. This means that, for the same velocity error, the errors in the calculated travel-time are smaller for long travel-times. The travel-time error for short travel-times (shallow events) is more sensitive to $E_{V_{p s}}$ than that for long travel-times (deep events). The travel-time error is also inversely proportional to the PS-wave velocity. High PS-wave velocity results in a small travel-time error. For a given travel-time, the error also depends on the location of the scatter point. A numerical analysis shows that the minimum value of $E_{V_{p s}}$ is reached at a special point which is close to the conversion point. The closer this point is to the location of the scatter point, the smaller $E_{V_{p s}}$. The values of $\gamma_{0}$ and $\gamma_{\text {eff }}$ have less effect on the travel-time error than the values of $V_{p s}$. The values of $\chi$ have a much smaller effect on the travel-time error than the effect of $\gamma_{0}, \gamma_{\text {eff }}$, and $V_{p s}$. Note that the location of the scatter point with the minimum $E_{V_{p s}}$ changes with $\gamma_{0}, \gamma_{e f f}$, and $V_{p s}$. The values of the travel-time error caused by $10 \mathrm{~m} / \mathrm{s}$ of velocity error for a $1000 \mathrm{~m} / \mathrm{s}$ velocity are at least $2 \mathrm{~ms}$ and can be more than $40 \mathrm{~ms}$. However, if the velocity is $2000 \mathrm{~m} / \mathrm{s}$, the travel-time errors are reduced to a quarter of these values.

Note that the travel-time error depends on the location of the scatter point. The travel time error becomes very large when the scatter point is at either end of a diffraction curve. Such scatter points should be eliminated. This can be achieved by aperture control during 
prestack Kirchhoff time migration processing. During PKTM, the aperture or the size of the diffraction is usually limited by a dip angle $\left[\beta=\arccos \left(\frac{t_{p s 0}}{t_{p s}}\right)\right]$ that acts as a dip limit filter. Data exceeding the dip limit is tapered to zero. But data that exceed the dip limit also have large travel-time errors. So tapering the data to zero eliminates data that have larger errors in travel-times. The dip limit angle can be alternatively determined by the travel-time error because the travel-time error can be calculated from the travel-time and the vertical traveltime of a $P S$-wave. Thus travel-time error provides an alternative criterion by which to determine the aperture angle.

Figure 4 shows absolute values of the travel-time error in the PS-wave caused by error of 0.1 in $\gamma_{0}$ (4\% of 2.5 and $2 \%$ of 4.5 ) with variation of $V_{p s}, \gamma_{0}, \gamma_{\text {eff }}, \chi$ and travel-times. In practice, this error is not small. Because $V_{p s}, \gamma_{e f f}$, and $\chi$ are correct, the travel-time error is $-E_{\gamma 0} \Delta \gamma_{0}$. All travel-time errors caused by errors in $\gamma_{0}$ are very small compared with the error caused by $V_{p s}$. This suggests that travel-time errors caused by errors in $\gamma_{0}$ can be neglected. The absolute values of the travel-time errors are also inversely proportional to the travel-time itself and to $V_{p s}$. Figure 4 also shows that small values of velocity ratios $\left(\gamma_{0}\right.$ and $\gamma_{\text {eff }}$ ) cause large errors in travel-time, and that large values of $\chi$ cause large errors in traveltime.

Figure 5 shows absolute values of the travel-time error in the PS-wave caused by an error of 0.1 in $\gamma_{\text {eff }}$ (5\% of 2.0 and $2.5 \%$ of 4.0) with variation of $V_{p s}, \gamma_{0}, \gamma_{\text {eff }}, \chi$ and traveltimes. In practice, this error is not small. Because $V_{p s}, \gamma_{0}$, and $\chi$ are correct, the travel-time error is $-E_{\text {reff }} \Delta \gamma_{\text {eff }}$. Note that the travel-time errors caused by errors in $\gamma_{\text {eff }}$ are far larger than the errors caused by similar variation in $\gamma_{0}$ in most causes. But in the cases with low velocity and short travel-time, the error in travel-time caused by errors in $\gamma_{\text {eff }}$ is less than that caused 
by errors in $\gamma_{0}$. The absolute values of the travel-time errors are also inversely proportional to the travel-time itself and to $V_{p s}$. However, the values of $\gamma_{0}, \gamma_{\text {eff }}, \chi$ have less effect on the travel-time error. All travel-time errors caused by error on $\gamma_{\text {eff }}$ change sign with the different locations of common image points. But the error is dominated by the error caused by the S-waves. This is because the error factor $E_{\text {res }}$ has a factor of $\frac{1}{2\left(1+\gamma_{\text {eff }}\right)}$ and whereas $E_{\text {reffp }}$ has a factor of $\frac{1}{2\left(1+\gamma_{\text {eff }}\right) \gamma_{\text {eff }}}$ which reduces the error in the P-waves. Note that the travel-time error depends on the location of the scatter point. The travel time errors become very large when the scatter point is at either end of a diffraction curve, especially at the end outside the source location. Such scatter points should be eliminated. This can be achieved by aperture control during prestack Kirchhoff time migration processing.

Figure 6 shows absolute values of the travel-time error in the PS-wave caused by an error of 0.05 in $\chi$ (50\% of 0.1 or $25 \%$ of 0.2$)$ with variation in $V_{p s}, \gamma_{0}, \gamma_{\text {eff }}, \chi$ and traveltimes. In practice, this error is not small. Because $V_{p s}, \gamma_{0}$ and $\gamma_{\text {eff }}$ are correct, the travel-time error is $-E_{\chi} \Delta \chi$. Figure 6 shows that travel time errors caused by errors in $\chi$ exhibits similar features to the travel-time error caused by error in $\gamma_{\text {eff }}$. For example, all travel-time errors caused by errors in $\chi$ changes sign with variation in CIP locations. The error is dominated by the error caused by the $\mathrm{S}$-waves. This is also because the error factor $E_{\chi s}$ has a factor of $\frac{1}{\left(\gamma_{0}-1\right)}$ whereas $E_{\chi p}$ has a factor of $\frac{1}{\left(\gamma_{0}-1\right) \gamma_{e f f}^{2}}$ which reduces the error in the P-waves.

The travel-time errors are also inversely proportional to the travel-time itself and to $V_{p s}$. The values of $\gamma_{0}, \gamma_{\text {eff }}, \chi$ have less effect on the travel-time error; the travel-time errors depend on the location of the scatter point and they become very large when the scatter point is at either end of a diffraction curve, especially at the end beyond the source location. We 
should also point out that an error of 0.05 is quite big for $\chi$ in weak anisotropic media. In practice, errors of $\chi$ are so small that errors in travel-time may be neglected.

\section{ANALYSIS OF EFFECTS OF PARAMETERS}

In this section, we compare the results for anisotropic media with the results for isotropic media obtained by Dai and Li (2006). The results for anisotropic media show similar features to the results for isotropic media. Firstly, for both anisotropic and isotropic media, errors in velocity have the largest effects on the travel-time error. The velocity ratio in both anisotropic and isotropic media has less effect on travel-time error. However, in anisotropic media, there are both a vertical velocity ratio and an effective velocity ratio. The vertical velocity ratio has much less effect on the travel-time error than the effective velocity ratio. The anisotropic parameter in anisotropic media has a similar effect to the effective velocity ratio. Secondly, the travel-time errors in both anisotropic and isotropic media are inversely proportional to the travel-time itself, which implies that the long travel-times are more accurately calculated than the short travel-times. Thirdly, the travel-time errors are inversely proportional to the PSwave velocity, which implies that the travel-time computation is more accurate in high velocity media than in low velocity media. The reason is that the error factors of PS wave velocity and effective velocity ratio are proportional to $w_{p}$ or $w_{s}$ and the error factor of anisotropic parameter is proportional to $w_{p}^{2}$ or $w_{s}^{2}$. Both $w_{p}$ or $w_{s}$ are inversely proportion to $V_{p s}^{2}$. Fourthly, the travel time error caused by the error in the velocity ratio in both anisotropic and isotropic media changes sign as the common image point locations vary. These sign changes result in asymmetric events in common image point gathers as we discussed in Dai and Li (2006). 
Since the travel-time error in anisotropic media has similar features as that in isotropic media, it has a similar effect on migration images. All discussions in Dai and Li (2006) can be applied to the case of anisotropic media and the same conclusions obtained. We will not repeat this analysis in this paper. This analysis shows that the velocity error mainly affects the position of the imaging point and velocity ratio error mainly affects the focusing of the imaging point. To obtain a focused image, the travel-time error must be less than some small value. To achieve this, the velocities must be as accurate as possible and a rough estimate of the P-to-S velocity ratio is needed.

The quality of the image is determined by the accuracy of the calculated travel-time. If there are no errors in the velocity model and the travel- times are correctly calculated, the energy will accumulate precisely at the desired image point. Otherwise, the energy will be smeared around the desired image point according to the errors in travel-times. Due to the energy smearing, the frequency of the resultant wavelet is lowered.

Because the error in travel-time caused by $\gamma_{0}$ is much less than that caused by $\gamma_{\text {eff }}$, $\chi$, and $V_{p s}$, we can ignore the effect of $\gamma_{0}$ on travel-time. That means we only need to consider the effects of $\gamma_{e f f}, \chi$, and $V_{p s}$ in migration velocity analysis and prestack time migration. $\gamma_{0}$ must be estimated by other methods.

To examine the effects of $\gamma_{\text {eff }}$ and $\chi$, two ratios are defined as:

$$
\frac{E_{\chi p}}{E_{\text {reffp }}}=\frac{2\left(1+\gamma_{\text {eff }}\right)}{\left(\gamma_{0}-1\right) \gamma_{\text {eff }}} \frac{w_{p}\left(1+w_{p}\right)}{\left\{1+2\left[1-2 \eta_{\text {eff }}\left(1+\gamma_{\text {eff }}\right)\right] w_{p}+\left[1-2 \eta_{\text {eff }}\left(1+2 \gamma_{\text {eff }}\right)\right] w_{p}^{2}\right\}},
$$

and

$$
\frac{E_{\chi s}}{E_{\text {reffs }}}=\frac{2\left(1+\gamma_{\text {eff }}\right)}{\left(\gamma_{0}-1\right)} \frac{w_{s}\left(1+w_{s}\right)}{\left[1+2\left(1+\xi_{\text {eff }}\right) w_{s}+\left(1-2 \xi_{\text {eff }}\right) w_{s}^{2}\right]} .
$$

Here $\Delta \gamma_{\text {eff }}=\Delta \chi$. The two ratios shows that, when $w_{p}$ and $w_{s}$ are small, $\chi$ has less effects on traveltime than $\gamma_{\text {eff }}$. However, when $w_{p}$ and $w_{s}$ are large, both ratios can be larger than 1 
which means $\chi$ has larger effects on traveltime than $\gamma_{\text {eff }}$. This can be explained by examining the differentials of the second and higher order moveout without considering the errors of $V_{p s}$ and $\gamma_{0}$. For P-waves raypath,

$$
\Delta w_{p}=\left[-\frac{w_{p}}{\left(1+\gamma_{\text {eff }}\right) \gamma_{\text {eff }}}\right] \Delta \gamma_{\text {eff }},
$$

and

$$
\Delta\left[\frac{\eta_{\text {eff }} w_{p}^{2}}{1+\left(1+2 \eta_{\text {eff }}\right) w_{p}}\right]=\frac{w_{p}^{2}\left(1+w_{p}\right)\left\{\Delta \chi-\chi\left[\frac{4+2 \gamma_{\text {eff }}+\left(3+2 \gamma_{\text {eff }}+2 \eta_{\text {eff }}\right) w_{p}}{\left(1+w_{p}\right)\left(1+\gamma_{\text {eff }}\right) \gamma_{\text {eff }}}\right] \Delta \gamma_{\text {eff }}\right\}}{\left[1+\left(1+2 \eta_{\text {eff }}\right) w_{p}\right]^{2}\left(\gamma_{0}-1\right) \gamma_{\text {eff }}^{2}} .
$$

Note in Equation 11, $\chi\left[\frac{4+2 \gamma_{\text {eff }}+\left(3+2 \gamma_{\text {eff }}+2 \eta_{\text {eff }}\right) w_{p}}{\left(1+w_{p}\right)\left(1+\gamma_{\text {eff }}\right) \gamma_{\text {eff }}}\right]$ decreases when $w_{p}$ increases and is less than $\chi\left[\frac{4+2 \gamma_{\text {eff }}}{\left(1+\gamma_{\text {eff }}\right) \gamma_{\text {eff }}}\right]$, which is far less than 1 , the effect of $\Delta \gamma_{\text {eff }}$ can be neglected. Equation 11 can be approximated as:

$$
\Delta\left[\frac{\eta_{\text {eff }} w_{p}^{2}}{1+\left(1+2 \eta_{\text {eff }}\right) w_{p}}\right] \approx \frac{w_{p}^{2}\left(1+w_{p}\right)}{\left[1+\left(1+2 \eta_{\text {eff }}\right) w_{p}\right]^{2}\left(\gamma_{0}-1\right) \gamma_{\text {eff }}^{2}} \Delta \chi
$$

For S-wave raypath,

$$
\Delta w_{s}=\left[\frac{w_{s}}{1+\gamma_{\text {eff }}}\right] \Delta \gamma_{\text {eff }}
$$

and

$$
\Delta\left(\frac{\xi_{\text {eff }} w_{s}^{2}}{1+w_{s}}\right)=-\frac{w_{s}^{2}}{\left(1+w_{s}\right)\left(\gamma_{0}-1\right)}\left[\Delta \chi+\chi \frac{2+w_{s}}{\left(1+w_{s}\right)\left(\gamma_{\text {eff }}+1\right)} \Delta \gamma_{\text {eff }}\right]
$$

Note In Equation 14, $\chi \frac{2+w_{s}}{\left(1+w_{s}\right)\left(\gamma_{\text {eff }}+1\right)}$ also decreases when $w_{s}$ increases and is far less than 1 . The effect of $\Delta \gamma_{\text {eff }}$ can be neglected. Equation 14 can be approximated as: 


$$
\Delta\left(\frac{\xi_{\text {eff }} w_{s}^{2}}{1+w_{s}}\right) \approx-\frac{w_{s}^{2}}{\left(1+w_{s}\right)\left(\gamma_{0}-1\right)} \Delta \chi .
$$

When $w_{p}$ and $w_{s}$ are small, $\Delta w_{p}$ is larger than $\Delta\left[\frac{\eta_{\text {eff }} w_{p}^{2}}{1+\left(1+2 \eta_{e f f}\right) w_{p}}\right]$ and $\Delta w_{s}$ is larger than $\Delta\left(\frac{\xi_{\text {eff }} w_{s}^{2}}{1+w_{s}}\right) \cdot \Delta \gamma_{\text {eff }}$ has larger effects on traveltime than $\Delta \chi$. When $w_{p}$ and $w_{s}$ are large, $\Delta w_{p}$ is less than $\Delta\left[\frac{\eta_{\text {eff }} w_{p}^{2}}{1+\left(1+2 \eta_{\text {eff }}\right) w_{p}}\right]$ and $\Delta w_{s}$ is less than $\Delta\left(\frac{\xi_{\text {eff }} w_{s}^{2}}{1+w_{s}}\right) . \Delta \chi$ has larger effects on traveltime than $\Delta \gamma_{\text {eff }}$.

The above analysis shows that when $w_{p}$ and $w_{s}$ are small, the contribution of $\Delta \chi$ can be neglected. However, when $w_{p}$ and $w_{s}$ are larger, the contribution of $\Delta \chi$ is larger than $\Delta \gamma_{\text {eff }}$.

The velocity error mainly affects the position of the imaging point whereas velocity ratio error and the anisotropic parameter mainly affect the focusing of the imaging point. The effect of $E_{V_{p s}}$ in mis-positioning is larger than that of $E_{\gamma 0}, E_{\text {reff }}$ and $E_{\chi}$. However, the effect of $E_{\gamma 0}, E_{\gamma e f f}$ and $E_{\chi}$ in focusing is larger than that of $E_{V p s}$. Note that the energy at a shallow image point has a larger travel-time error than that at a deep image point. This explains why the quality of the shallow part of the migrated image is poorer than the deep part.

For dipping targets, the effect of error in $\gamma_{\text {eff }}$ and $\chi$ increases with increasing dip angle of a target. The larger the dip angle, the larger the effect of the error in $\gamma_{\text {eff }}$ and $\chi$. The events in CIP gathers appear asymmetric due to the error in $\gamma_{\text {eff }}$ and $\chi$. 
The above conclusions have been used to guide how to update the migration velocity model for real seismic dataset (Dai and Li, 2007a, b). In these two papers, both synthetic and real examples confirm the above conclusions.

\section{CONCLUSIONS}

We have investigated the factors that affect the travel-time accuracy of $P S$-waves calculated in prestack Kirchhoff time migration and the effects on the prestack migrated image for PSwaves in anisotropic media. The travel-time error in a PS-wave is the sum of the products of the relative errors in $V_{p s}, \gamma_{e f f}, \gamma_{0}$, and $\chi$ and their corresponding error factors $E_{V_{p s}}, E_{\gamma 0}$, $E_{\text {reff }}$ and $E_{\chi}$. These error factors modulate the effects of $V_{p s}, \gamma_{\text {eff }}, \gamma_{0}$, and $\chi$ on the traveltime computation.

Theoretical and numerical analyses of the error factors show that all the error factors are inversely proportional to the velocity as well as to the travel-time. However, the traveltime of a PS-wave is affected more severely by error in $V_{p s}$ than by error in $\gamma_{\text {eff }}, \gamma_{0}$, and $\chi$. The error in $V_{p s}$ causes the largest error in the travel-time and the error in $\gamma_{0}$ causes the smallest error in the travel time. The error in $\gamma_{\text {eff }}$ causes a larger error in the travel-time than error in $\chi$ when the ratio of horizontal distance to depth $\left(w_{p}\right.$ or $\left.w_{s}\right)$ is small. However, for far-offset data, The error in $\chi$ causes a larger error in the travel-time than error in $\gamma_{\text {eff }}$ when the ratio of horizontal distance to depth. Errors caused by $\gamma_{\text {eff }}$ and $\chi$ are mainly from the converted S-wave raypath of PS converted waves. This conclusion is useful for processing PS converted wave data with weak anisotropy using prestack Kirchhoff time migration. Because the travel time is very sensitive to $V_{p s}$, we must accurately estimate $V_{p s}$. However the fact that the travel time is less sensitive to $\gamma_{0}, \gamma_{\text {eff }}$ and $\chi$, is convenient since we may not able to 
accurately estimate them. Hence roughly estimated $\gamma_{0}, \gamma_{\text {eff }}$ and $\chi$ can be used in PSTM. In practice, we can use values of $\gamma_{0}, \gamma_{\text {eff }}$ and $\chi$ obtained from stacking velocity analysis. This can save processing cost and time. Errors in travel-time for shallow events are usually larger than those for deep events. This may explain why the quality of the shallow part of a migrated image is poorer than that of the deep part. The effect of velocity ratio error depends on the dip of a target. More accurate $\gamma_{\text {eff }}$ and $\chi$ values is necessary for migrating dipping targets. These findings are also useful for our understanding of PS-wave behaviour and for $P S$-wave imaging in anisotropic media.

\section{ACKNOWLEDGEMENTS}

This work is funded by the Edinburgh Anisotropy Project (EAP) of the British Geological Survey, and is published with the permission of the Executive Director of the British Geological Survey (NERC) and the EAP sponsors: BG, BGP, BP, Chevron, ConocoPhillips, CNPC, ENI-Agip, ExxonMobil, GX Technology, Landmark, Marathon, Norsk Hydro, PDVSA, Schlumberger, Shell, SinoPec, Total, and Veritas.

\section{Appendix A: Parameters of equivalent anisotropic medium}

Consider an $N$-layer vertical-transverse-isotropic medium with interval Thomsen's parameters $\left(V_{p 0 i}, \varepsilon_{i}, V_{s 0 i}, \delta_{i}\right.$, and $\left.z_{i}, i=1,2,3, \ldots, n\right)$ for each layer (Figure 7). The following parameters are defined: The short-spread normal moveout velocity for P-waves and S-waves and effective anisotropy parameters are (Tsvankin and Thomsen,1994):

$$
V_{p 2 i}=V_{p 0 i} \sqrt{\left(1+2 \delta_{i}\right.} \text {. }
$$




$$
V_{s 2 i}=V_{s 0 i} \sqrt{\left(1+2 \sigma_{i}\right.} \text {. }
$$

where

$$
\sigma_{i}=\frac{V_{p 0 i}^{2}}{V_{s 0 i}^{2}}\left(\varepsilon_{i}-\delta_{i}\right)
$$

The anisotropy parameters for P-waves and S-waves are:

$$
\begin{gathered}
\eta_{i}=\frac{\varepsilon_{i}-\delta_{i}}{\left(1+2 \delta_{i}\right)^{2}}\left(1+\frac{2 \delta_{i}}{1-V_{s 0 i}^{2} / V_{p 0 i}^{2}}\right) . \\
\zeta_{i}=\frac{\sigma_{i}}{\left(1+2 \sigma_{i}\right)^{2}}\left(1+\frac{2 \delta_{i}}{1-V_{s 0 i}^{2} / V_{p 0 i}^{2}}\right) .
\end{gathered}
$$

Note from the above definition, we have $\xi_{i}=\gamma_{\text {effi }}^{2} \eta_{i}$. This $N$-layer vertical-transverseisotropic medium can be considered as an equivalent anisotropic medium. Based on the above parameters, we can define the following effective parameters of this equivalent medium for P, S, and PS converted waves:

$$
\begin{aligned}
& t_{p 0}=\sum_{i=1}^{n} t_{p 0 i}, \\
& t_{s 0}=\sum_{i=1}^{n} t_{s 0 i}, \\
& t_{p s 0}=\sum_{i=1}^{n} t_{p 0 i}+\sum_{i=1}^{n} t_{s 0 i}=t_{p 0}+t_{s 0}
\end{aligned}
$$

are the vertical traveltimes for the P-, S- and PS converted wave respectively;

$$
\gamma_{0}=\frac{t_{s 0}}{t_{p 0}}
$$

is the vertical velocity ratio; 


$$
\begin{aligned}
& V_{p 2}^{2}=\frac{1}{t_{p 0}} \sum_{i=1}^{n} V_{p 2 i}^{2} t_{p 0 i}, \\
& V_{s 2}^{2}=\frac{1}{t_{s 0}} \sum_{i=1}^{n} V_{s 2 i}^{2} t_{s 0 i},
\end{aligned}
$$

and

$$
V_{p s 2}^{2}=\frac{1}{t_{c 0}}\left[\sum_{i=1}^{n} V_{p 2 i}^{2} t_{p 0 i}+\sum_{i=1}^{n} V_{s 2 i}^{2} t_{s 0 i}\right]
$$

are the squares of the stacking velocities for P-, S-, and PS converted waves, respectively;

$$
\gamma_{e f f}=\frac{1}{\gamma_{0}} \frac{V_{p 2}^{2}}{V_{s 2}^{2}}
$$

is the effective velocity ratio. Note that although $\gamma_{\text {eff }}$ is defined by $\gamma_{0}, V_{p 2}$ and $V_{s 2}$, the change in $\gamma_{0}$ does not necessarily to change $\gamma_{\text {eff }}$ value because it change the value of $\frac{V_{p 2}}{V_{s 2}}$. The change in $\gamma_{\text {eff }}$ will affect the value of $\frac{V_{p 2}}{V_{s 2}}$ either, not $\gamma_{0}$. So we can treat $\gamma_{\text {eff }}$ and $\gamma_{0}$ as independent parameters in estimation procedure.

$$
\begin{gathered}
\eta_{\text {eff }}=\frac{1}{8 t_{p 0} V_{p 2}^{4}}\left[\sum_{i=1}^{n} V_{p 2 i}^{4}\left(1+8 \eta_{i}\right) \Delta t_{p 0 i}-t_{p 0} V_{p 2}^{4}\right], \\
\zeta_{\text {eff }}=\frac{1}{8 t_{s 0} V_{s 2}^{4}}\left[\sum_{i=1}^{n} V_{s 2 i}^{4}\left(1-8 \zeta_{i}\right) \Delta t_{s 0 i}-t_{s 0} V_{s 2}^{4}\right]
\end{gathered}
$$

are the anisotropic coefficients for P- and S-waves respectively; and

$$
\chi_{\text {eff }}=\gamma_{0} \gamma_{\text {eff }}^{2} \eta_{\text {eff }}+\zeta_{\text {eff }}
$$

is the anisotropic coefficient for PS converted waves. Note that we change the sign of $\zeta_{\text {eff }}$ for convenience.

Note that the parameters for PS converted waves can be converted to parameters for $\mathrm{P}$ and S waves or vice versa. 


$$
\begin{gathered}
t_{p 0}=\frac{t_{p s 0}}{1+\gamma_{0}} \\
t_{s 0}=\frac{\gamma_{0} t_{p s 0}}{1+\gamma_{0}} \\
v_{p 2}^{2}=v_{p s 2}^{2} \frac{\gamma_{\text {eff }}\left(1+\gamma_{0}\right)}{1+\gamma_{\text {eff }}} \\
v_{s 2}^{2}=v_{p s 2}^{2} \frac{\left(1+\gamma_{0}\right)}{\left(1+\gamma_{\text {eff }}\right) \gamma_{0}} \\
\xi_{\text {eff }}=-\eta_{\text {eff }} \gamma_{\text {eff }}^{2} \\
\chi_{\text {eff }}=\eta_{\text {eff }} \gamma_{0} \gamma_{\text {eff }}^{2}+\xi_{\text {eff }}=\left(\gamma_{0}-1\right) \gamma_{\text {eff }}^{2} \eta_{\text {eff }}
\end{gathered}
$$

The P- and S- waves are reflected at the bottom of the $N$-th layer. The PS converted wave is converted at the conversion point of the $N$-th layer with a down-going P-leg and an up-going S-leg. Their moveouts are approximately written with effective parameters using Thomsen's notation (1999) as (Alkhalifah, 1997; Li and Yuan 2003):

$$
\begin{aligned}
t_{p s} & =t_{p}+t_{s}, \\
t_{p} & =\sqrt{t_{p 0}^{2}+\frac{x_{p}^{2}}{V_{p 2}^{2}}-2 \eta_{\text {eff }} \frac{x_{p}^{4}}{V_{p 2}^{2}\left[t_{p 0}^{2} V_{p 2}^{2}+\left(1+2 \eta_{e f f}\right) x_{p}^{2}\right]}}, \\
t_{s} & =\sqrt{t_{s 0}^{2}+\frac{x_{s}^{2}}{V_{s 2}^{2}}-2 \xi_{\text {eff }} \frac{x_{s}^{4}}{V_{s 2}^{2}\left(t_{s 0}^{2} V_{s 2}^{2}+x_{s}^{2}\right)}},
\end{aligned}
$$

where $x_{p}$ is the distance between the source and conversion point and $x_{s}$ is the distance between the conversion point and receiver.

Let $\quad w_{p}=\frac{x_{p}^{2}\left(1+\gamma_{0}\right)\left(1+\gamma_{\text {eff }}\right)}{t_{p s 0}^{2} V_{p s}^{2} \gamma_{\text {eff }}} \quad, \quad w_{s}=\frac{x_{s}^{2}\left(1+\gamma_{0}\right)\left(1+\gamma_{\text {eff }}\right)}{t_{p s 0}^{2} V_{p s}^{2} \gamma_{0}}, \quad \eta_{\text {eff }}=\frac{\chi_{\text {eff }}}{\left(\gamma_{0}-1\right) \gamma_{\text {eff }}^{2}} \quad, \quad$ and $\xi_{\text {eff }}=-\frac{\chi_{\text {eff }}}{\gamma_{0}-1}$, we have: 


$$
\begin{aligned}
& t_{p}=\frac{t_{p s 0}}{1+\gamma_{0}} \sqrt{1+w_{p}-\frac{2 \eta_{\text {eff }}}{1+\left(1+2 \eta_{\text {eff }}\right) w_{p}} w_{p}^{2}} \\
& t_{s}=\frac{\gamma_{0} t_{p s 0}}{1+\gamma_{0}} \sqrt{1+w_{s}-\frac{2 \xi_{\text {eff }}}{1+w_{s}} w_{s}^{2}}
\end{aligned}
$$

\section{Appendix B: The error factor of travel time of prestack Kirchhoff time migration.}

The error in the traveltime of the PS converted wave in prestack Kirchhoff time migration is

$$
\Delta t_{p s}=\Delta t_{p}+\Delta t_{s}
$$

Here

$$
\Delta t_{p}=\Delta\left\{\frac{t_{p s 0}}{1+\gamma_{0}} \sqrt{1+w_{p}-\frac{2 \eta_{\text {eff }}}{1+\left(1+2 \eta_{\text {eff }}\right) w_{p}} w_{p}^{2}}\right\},
$$

and

$$
\Delta t_{s}=\Delta\left\{\frac{\gamma_{0} t_{p s 0}}{1+\gamma_{0}} \sqrt{1+w_{s}-\frac{2 \xi_{\text {eff }}}{1+w_{s}} w_{s}^{2}}\right\} .
$$

Equations (B2) and (B3) can be written as:

$$
\begin{aligned}
& \Delta t_{p}=\left\{-\frac{\Delta \gamma_{0}}{1+\gamma_{0}}+\frac{\left[1+2 w_{p}+\left(1+2 \eta_{\text {eff }}\right) w_{p}^{2}\right] \Delta w_{p}-2\left(1+w_{p}\right) w_{p}^{2} \Delta \eta_{\text {eff }}}{2\left[1+\left(1+2 \eta_{\text {eff }}\right) w_{p}\right]\left[1+2\left(1+\eta_{\text {eff }}\right) w_{p}+w_{p}^{2}\right]} t_{p}\right. \\
& \Delta t_{s}=\left\{\frac{\Delta \gamma_{0}}{\left(1+\gamma_{0}\right) \gamma_{0}}+\frac{\left[\left(1+w_{s}\right)^{2}-2\left(2+w_{s}\right) w_{s} \xi_{\text {eff }}\right] \Delta w_{s}-2 w_{s}^{2}\left(1+w_{s}\right) \Delta \xi_{\text {eff }}}{2\left[\left(1+2 w_{s}+w_{s}^{2}\left(1-2 \xi_{\text {eff }}\right)\right]\left(1+w_{s}\right)\right.}\right\} t_{s}
\end{aligned}
$$

Because $\quad w_{p}=\frac{x_{p}^{2}\left(1+\gamma_{0}\right)\left(1+\gamma_{e f f}\right)}{t_{p s 0}^{2} V_{p s}^{2} \gamma_{e f f}}, \quad w_{s}=\frac{x_{s}^{2}\left(1+\gamma_{0}\right)\left(1+\gamma_{\text {eff }}\right)}{t_{p s 0}^{2} V_{p s}^{2} \gamma_{0}}, \quad \eta_{\text {eff }}=\frac{\chi_{\text {eff }}}{\left(\gamma_{0}-1\right) \gamma_{\text {eff }}^{2}}, \quad$ and $\xi_{\text {eff }}=-\frac{\chi_{\text {eff }}}{\gamma_{0}-1}$, their differentials are written as: 


$$
\begin{gathered}
\Delta w_{p}=\left[\frac{\Delta \gamma_{0}}{1+\gamma_{0}}-\frac{\Delta \gamma_{\text {eff }}}{\left(1+\gamma_{\text {eff }}\right) \gamma_{\text {eff }}}-2 \frac{\Delta V_{p s}}{V_{p s}}\right] w_{p}, \\
\Delta \eta_{\text {eff }}=\left[\frac{\Delta \chi_{\text {eff }}}{\chi_{\text {eff }}}-\frac{\Delta \gamma_{0}}{1+\gamma_{0}}-2 \frac{\Delta \gamma_{\text {eff }}}{\gamma_{\text {eff }}}\right] \eta_{\text {eff }}, \\
\Delta w_{s}=\left[-\frac{\Delta \gamma_{0}}{\left(1+\gamma_{0}\right) \gamma_{0}}+\frac{\Delta \gamma_{\text {eff }}}{1+\gamma_{\text {eff }}}-2 \frac{\Delta V_{p s}}{V_{p s}}\right] w_{s},
\end{gathered}
$$

and

$$
\Delta \xi_{\text {eff }}=\left[\frac{\Delta \chi_{\text {eff }}}{\chi_{\text {eff }}}-\frac{\Delta \gamma_{0}}{1+\gamma_{0}}\right] \xi_{\text {eff }}
$$

Note that the above partial differentiations are only applied when the errors in the four parameters are small. This assumption is easily assured. For example, if the initial velocity model has large errors, we can repeat the velocity analysis to reduce the errors.

Substituting them in Equations B-4 and B-5, we have

$$
\begin{gathered}
{\left[1+2 w_{p}+\left(1+2 \eta_{\text {eff }}\right) w_{p}^{2}\right]\left[\frac{\Delta \gamma_{0}}{1+\gamma_{0}}-\frac{\Delta \gamma_{\text {eff }}}{\left(1+\gamma_{\text {eff }}\right) \gamma_{\text {eff }}}-2 \frac{\Delta V_{p s}}{V_{p s}}\right] w_{p}} \\
\Delta t_{p}=\left\{-\frac{\Delta \gamma_{0}}{1+\gamma_{0}}+\frac{-2\left(1+w_{p}\right) w_{p}^{2}\left[\frac{\Delta \chi_{\text {eff }}}{\chi_{\text {eff }}}-\frac{\Delta \gamma_{0}}{1+\gamma_{0}}-2 \frac{\Delta \gamma_{\text {eff }}}{\gamma_{\text {eff }}}\right] \eta_{\text {eff }}}{2\left[1+\left(1+2 \eta_{\text {eff }}\right) w_{p}\right]\left[1+2\left(1+\eta_{\text {eff }}\right) w_{p}+w_{p}^{2}\right]}\right\} t_{p} \\
\Delta t_{s}=\left\{\frac{\Delta \gamma_{0}}{\left(1+\gamma_{0}\right) \gamma_{0}}+\frac{-2 w_{s}^{2}\left(1+w_{s}\right)\left[\frac{\Delta \chi_{\text {eff }}}{\chi_{\text {eff }}}-\frac{\Delta \gamma_{0}}{1+\gamma_{0}}\right] \xi_{\text {eff }}}{2\left[\left(1+2 w_{s}+w_{s}^{2}\left(1-2 \xi_{\text {eff }}\right)\right]\left(1+w_{s}\right)\right.}-\frac{\Delta \gamma_{0}}{\left(1+\gamma_{0}\right) \gamma_{0}}+\frac{\Delta \gamma_{\text {eff }}}{1+\gamma_{\text {eff }}}-2 \frac{\Delta V_{p s}}{V_{p s}}\right] w_{s}
\end{gathered}
$$

Putting Equation B-10 and B-11 together and rearranging items, we obtained Equation 4, 5, 6,7 , and 8 .

\section{REFERENCES}


Alkhalifah T., 1997, Velocity analysis using nonhyperbolic moveout in transversely isotropic media: Geophysics 62, 1839-1854.

Dai, H., and Li, X.-Y., 2007a, Velocity model updating in prestack Kirchhoff time migration for PS converted waves: Part I - Theory, Geophysical Prospecting, 55, 525-547

Dai, H., and Li, X.-Y., 2007b, Velocity model updating in prestack Kirchhoff time migration for PS converted waves: Part II - Application, Geophysical Prospecting, 55, 549-559

Dai, H. and Li, X-Y., 2006, The effect of migration velocity errors on traveltime accuracy in prestack Kirchhoff time migration and the image of PS converted waves: Geophysics 71, No2, S73-S83.

Li, X.-Y. and Crampin, S., 1993, Approximations to shear-wave velocity and moveout equations in anisotropic media: Geophysical Prospecting, 41, 833-857

Li, X.-Y., Dai, H., Mancini, F. 2007, Converted-wave imaging in anisotropic media: theory and case studies: Geophysical Prospecting, 55, 345-363

Li, X-Y. and Yuan, J., 2003, Converted-wave moveout and conversion-point equations in layered VTI media: theory and applications: Journal of Applied Geophysics 54, 297-318.

Thomsen L., 1999, Converted wave reflection seismology over inhomogeneous, anisotropic media: Geophysics 64, 678-690.

Tsvankin, I. and Thomsen, L., 1994, Nonhyperbolic reflection moveout in anisotropic media: Geophysics, 59, 1290-1304. 


\section{Caption list}

Figure 1. The relationship between the source, receiver and scatter point.

Figure 2. Diffraction curves calculated using various velocities and velocity ratios in prestack time migration. (a) $\mathrm{V}_{\mathrm{ps}}=1000 \mathrm{~m} / \mathrm{s}, \gamma_{0}=2.5, \gamma_{\mathrm{eff}}=2.0, \chi_{\mathrm{eff}}=0.1$; (b) $\mathrm{V}_{\mathrm{ps}}=1000 \mathrm{~m} / \mathrm{s}, \gamma_{0}=2.5, \gamma_{\mathrm{eff}}=2.0$, $\chi_{\text {eff }}=0.2$; (c) $V_{\mathrm{ps}}=1000 \mathrm{~m} / \mathrm{s}, \gamma_{0}=4.5, \gamma_{\mathrm{eff}}=4.0, \chi_{\mathrm{eff}}=0.1$; (d) $\mathrm{V}_{\mathrm{ps}}=1000 \mathrm{~m} / \mathrm{s}, \gamma_{0}=4.5, \gamma_{\mathrm{eff}}=4.0$, $\chi_{\text {eff }}=0.2$; (e) $V_{\mathrm{ps}}=2000 \mathrm{~m} / \mathrm{s}, \gamma_{0}=2.5, \gamma_{\mathrm{eff}}=2.0, \chi_{\mathrm{eff}}=0.1$; (f) $\mathrm{V}_{\mathrm{ps}}=2000 \mathrm{~m} / \mathrm{s}, \gamma_{0}=2.5, \gamma_{\mathrm{eff}}=2.0$, $\chi_{\text {eff }}=0.2$; (g) $V_{\mathrm{ps}}=2000 \mathrm{~m} / \mathrm{s}, \gamma_{0}=4.5, \gamma_{\mathrm{eff}}=4.0, \chi_{\mathrm{eff}}=0.1$; (h) $\mathrm{V}_{\mathrm{ps}}=2000 \mathrm{~m} / \mathrm{s}, \gamma_{0}=4.5, \gamma_{\mathrm{eff}}=4.0$, $\chi_{\text {eff }}=0.2$. The source is at 0 and receiver is at 1.0 of the CIP (Common image gather) location. The CIP location is defined as offset/1000m. Five curves show the vertical two-way travel time as functions of CIP location for different travel-times (solid: $t_{p s}=1.0 \mathrm{~s}$; dot: $t_{p s}=2.0 \mathrm{~s}$; short dash: $t_{p s}=3.0 \mathrm{~s}$; middle dash: $t_{p s}=4.0 \mathrm{~s}$; long dash: $t_{p s}=5.0 \mathrm{~s}$ ).

Figure 3. The time error related to $E_{V_{p s}}$ caused by $10 \mathrm{~ms}(1 \%$ of $1000 \mathrm{~m} / \mathrm{s}$ or $0.5 \%$ of $2000 \mathrm{~m} / \mathrm{s})$ error in $V_{p s}$. Five curves show the factor values against the CIP location with different traveltimes as specified in Figure 2. (a) $\mathrm{V}_{\mathrm{ps}}=1000 \mathrm{~m} / \mathrm{s}, \gamma_{0}=2.5, \gamma_{\mathrm{eff}}=2.0, \chi_{\mathrm{eff}}=0.1$; (b) $\mathrm{V}_{\mathrm{ps}}=1000 \mathrm{~m} / \mathrm{s}$, $\gamma_{0}=2.5, \gamma_{\mathrm{eff}}=2.0, \chi_{\mathrm{eff}}=0.2$; (c) $\mathrm{V}_{\mathrm{ps}}=1000 \mathrm{~m} / \mathrm{s}, \gamma_{0}=4.5, \gamma_{\mathrm{eff}}=4.0, \chi_{\mathrm{eff}}=0.1$; (d) $\mathrm{V}_{\mathrm{ps}}=1000 \mathrm{~m} / \mathrm{s}$, $\gamma_{0}=4.5, \gamma_{\mathrm{eff}}=4.0, \chi_{\mathrm{eff}}=0.2$; (e) $\mathrm{V}_{\mathrm{ps}}=2000 \mathrm{~m} / \mathrm{s}, \gamma_{0}=2.5, \gamma_{\mathrm{eff}}=2.0, \chi_{\mathrm{eff}}=0.1$; (f) $\mathrm{V}_{\mathrm{ps}}=2000 \mathrm{~m} / \mathrm{s}$, $\gamma_{0}=2.5, \gamma_{\mathrm{eff}}=2.0, \chi_{\mathrm{eff}}=0.2$; (g) $\mathrm{V}_{\mathrm{ps}}=2000 \mathrm{~m} / \mathrm{s}, \gamma_{0}=4.5, \gamma_{\mathrm{eff}}=4.0, \chi_{\mathrm{eff}}=0.1$; (h) $\mathrm{V}_{\mathrm{ps}}=2000 \mathrm{~m} / \mathrm{s}$, $\gamma_{0}=4.5, \gamma_{\mathrm{eff}}=4.0, \chi_{\mathrm{eff}}=0.2$.

Figure 4 . The time error related to $E_{\gamma_{0}}$ caused by 0.1 ( $4 \%$ of 2.5 or $2 \%$ of 4.5 ) error in $\gamma_{0}$. Five curves show the factor values against the CIP location with different travel-times as 
specified in Figure 2. (a) $\mathrm{V}_{\mathrm{ps}}=1000 \mathrm{~m} / \mathrm{s}, \gamma_{0}=2.5, \gamma_{\mathrm{eff}}=2.0, \chi_{\mathrm{eff}}=0.1$; (b) $\mathrm{V}_{\mathrm{ps}}=1000 \mathrm{~m} / \mathrm{s}, \gamma_{0}=2.5$, $\gamma_{\mathrm{eff}}=2.0, \chi_{\mathrm{eff}}=0.2$; (c) $\mathrm{V}_{\mathrm{ps}}=1000 \mathrm{~m} / \mathrm{s}, \gamma_{0}=4.5, \gamma_{\mathrm{eff}}=4.0, \chi_{\mathrm{eff}}=0.1$; (d) $\mathrm{V}_{\mathrm{ps}}=1000 \mathrm{~m} / \mathrm{s}, \gamma_{0}=4.5$, $\gamma_{\mathrm{eff}}=4.0, \chi_{\mathrm{eff}}=0.2$; (e) $\mathrm{V}_{\mathrm{ps}}=2000 \mathrm{~m} / \mathrm{s}, \gamma_{0}=2.5, \gamma_{\mathrm{eff}}=2.0, \chi_{\mathrm{eff}}=0.1$; (f) $\mathrm{V}_{\mathrm{ps}}=2000 \mathrm{~m} / \mathrm{s}, \gamma_{0}=2.5$, $\gamma_{\mathrm{eff}}=2.0, \chi_{\mathrm{eff}}=0.2 ;$ (g) $\mathrm{V}_{\mathrm{ps}}=2000 \mathrm{~m} / \mathrm{s}, \gamma_{0}=4.5, \gamma_{\mathrm{eff}}=4.0, \chi_{\mathrm{eff}}=0.1$; (h) $\mathrm{V}_{\mathrm{ps}}=2000 \mathrm{~m} / \mathrm{s}, \gamma_{0}=4.5$, $\gamma_{\mathrm{eff}}=4.0, \chi_{\mathrm{eff}}=0.2$

Figure 5. The time error related to $E_{\gamma_{\text {eff }}}$ caused by 0.1 (5\% of 2.0 or $2.5 \%$ of 4.0 ) error in $\gamma_{\text {eff }}$. Five curves show the factor values against the CIP location with different travel-times as specified in Figure 2. (a) $V_{\mathrm{ps}}=1000 \mathrm{~m} / \mathrm{s}, \gamma_{0}=2.5$, $\gamma_{\mathrm{eff}}=2.0, \chi_{\text {eff }}=0.1$; (b) $\mathrm{V}_{\mathrm{ps}}=1000 \mathrm{~m} / \mathrm{s}, \gamma_{0}=2.5$, $\gamma_{\mathrm{eff}}=2.0, \chi_{\mathrm{eff}}=0.2 ;$ (c) $\mathrm{V}_{\mathrm{ps}}=1000 \mathrm{~m} / \mathrm{s}, \gamma_{0}=4.5, \gamma_{\mathrm{eff}}=4.0, \chi_{\mathrm{eff}}=0.1$; (d) $\mathrm{V}_{\mathrm{ps}}=1000 \mathrm{~m} / \mathrm{s}, \gamma_{0}=4.5$, $\gamma_{\mathrm{eff}}=4.0, \chi_{\mathrm{eff}}=0.2$; (e) $\mathrm{V}_{\mathrm{ps}}=2000 \mathrm{~m} / \mathrm{s}, \gamma_{0}=2.5, \gamma_{\mathrm{eff}}=2.0, \chi_{\mathrm{eff}}=0.1$; (f) $\mathrm{V}_{\mathrm{ps}}=2000 \mathrm{~m} / \mathrm{s}, \gamma_{0}=2.5$, $\gamma_{\mathrm{eff}}=2.0, \chi_{\mathrm{eff}}=0.2 ;$ (g) $\mathrm{V}_{\mathrm{ps}}=2000 \mathrm{~m} / \mathrm{s}, \gamma_{0}=4.5, \gamma_{\mathrm{eff}}=4.0, \chi_{\mathrm{eff}}=0.1$; (h) $\mathrm{V}_{\mathrm{ps}}=2000 \mathrm{~m} / \mathrm{s}, \gamma_{0}=4.5$, $\gamma_{\mathrm{eff}}=4.0, \chi_{\mathrm{eff}}=0.2$

Figure 6. The time error related to $E_{\chi}$ caused by 0.05 (50\% of 0.1 or $25 \%$ of 0.2 ) error in $\chi$. Five curves show the factor values against the CIP location with different travel-times as specified in Figure 2. (a) $\mathrm{V}_{\mathrm{ps}}=1000 \mathrm{~m} / \mathrm{s}, \gamma_{0}=2.5, \gamma_{\mathrm{eff}}=2.0, \chi_{\mathrm{eff}}=0.1$; (b) $\mathrm{V}_{\mathrm{ps}}=1000 \mathrm{~m} / \mathrm{s}, \gamma_{0}=2.5$, $\gamma_{\mathrm{eff}}=2.0, \chi_{\mathrm{eff}}=0.2 ;$ (c) $\mathrm{V}_{\mathrm{ps}}=1000 \mathrm{~m} / \mathrm{s}, \gamma_{0}=4.5, \gamma_{\mathrm{eff}}=4.0, \chi_{\mathrm{eff}}=0.1$; (d) $\mathrm{V}_{\mathrm{ps}}=1000 \mathrm{~m} / \mathrm{s}, \gamma_{0}=4.5$, $\gamma_{\mathrm{eff}}=4.0, \chi_{\mathrm{eff}}=0.2$; (e) $\mathrm{V}_{\mathrm{ps}}=2000 \mathrm{~m} / \mathrm{s}, \gamma_{0}=2.5, \gamma_{\mathrm{eff}}=2.0, \chi_{\mathrm{eff}}=0.1$; (f) $\mathrm{V}_{\mathrm{ps}}=2000 \mathrm{~m} / \mathrm{s}, \gamma_{0}=2.5$, $\gamma_{\text {eff }}=2.0, \chi_{\text {eff }}=0.2 ;$ (g) $V_{\mathrm{ps}}=2000 \mathrm{~m} / \mathrm{s}, \gamma_{0}=4.5, \gamma_{\mathrm{eff}}=4.0, \chi_{\mathrm{eff}}=0.1$; (h) $\mathrm{V}_{\mathrm{ps}}=2000 \mathrm{~m} / \mathrm{s}, \gamma_{0}=4.5$, $\gamma_{\mathrm{eff}}=4.0, \chi_{\mathrm{eff}}=0.2$

Figure 7. Multi-layer anisotropic media and the ray path of P, S and PS converted waves. 


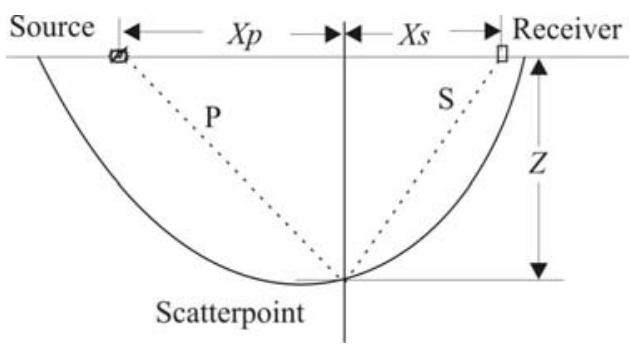

Figure 1. 
(a)

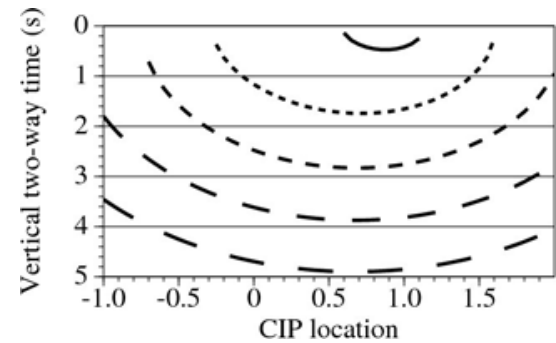

(c)

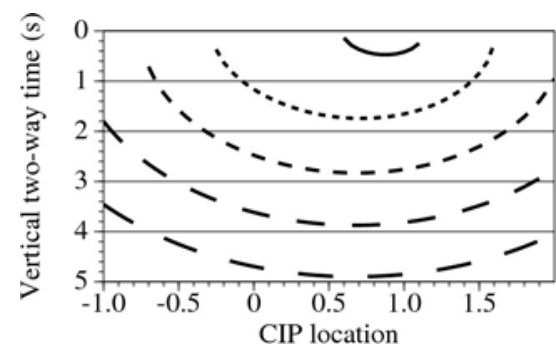

(e)

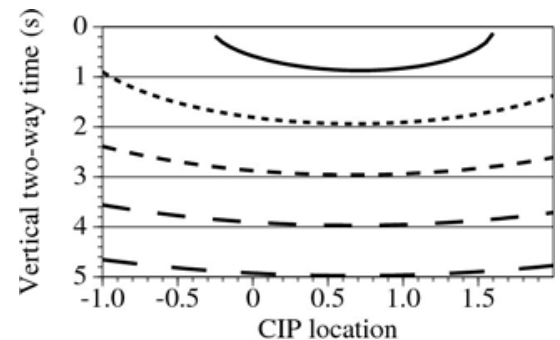

(g)

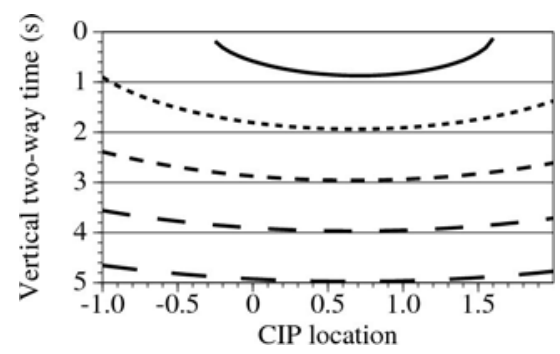

(b)

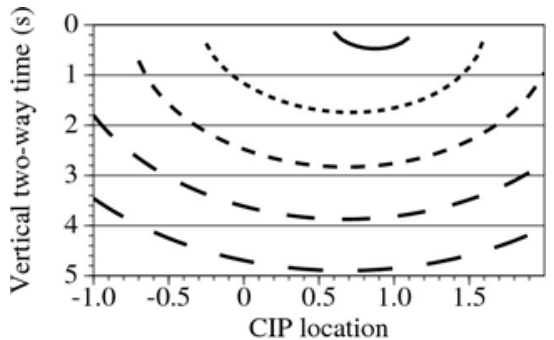

(d)

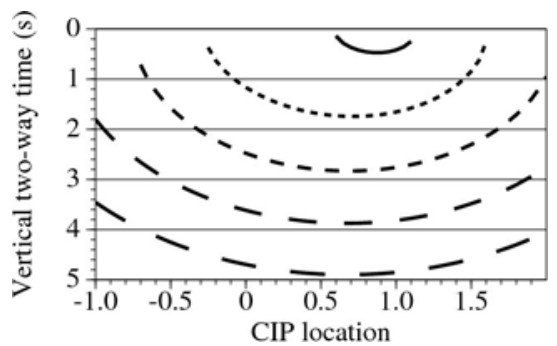

(f)

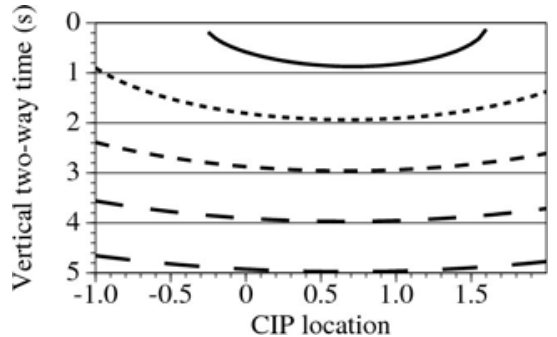

(h)

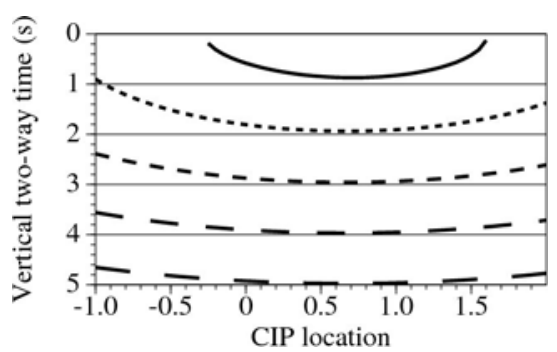

Figure 2. 
(a)

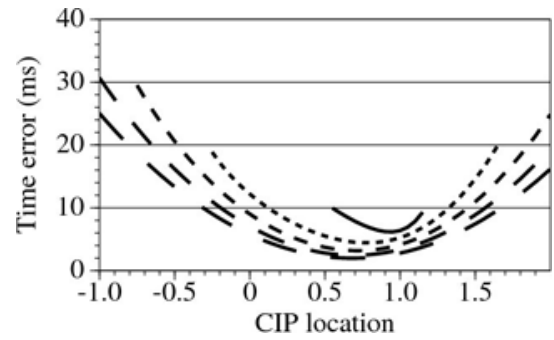

(c)

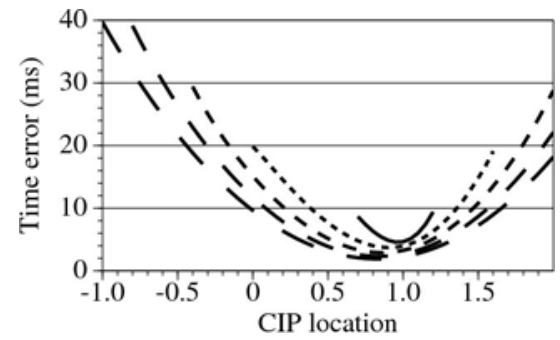

(e)

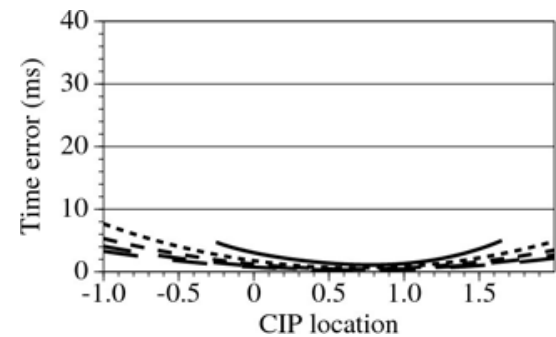

(g)

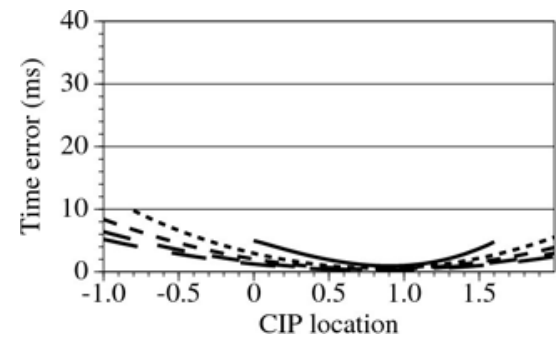

(b)

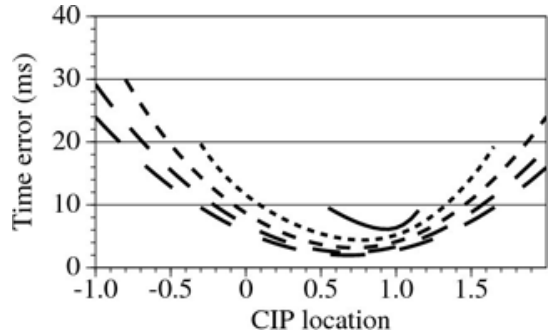

(d)

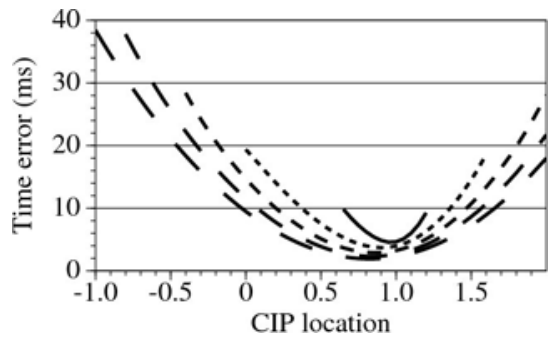

(f)

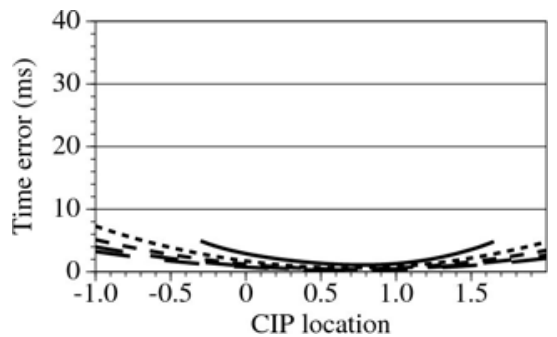

(h)

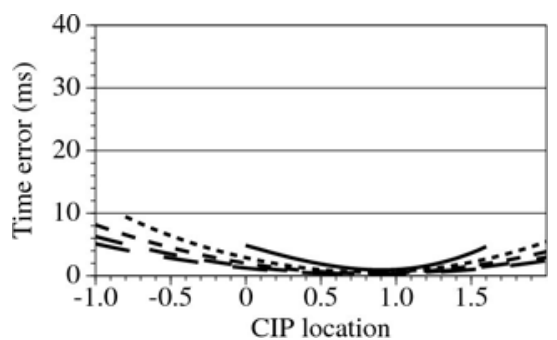

Figure 3. 
(a)

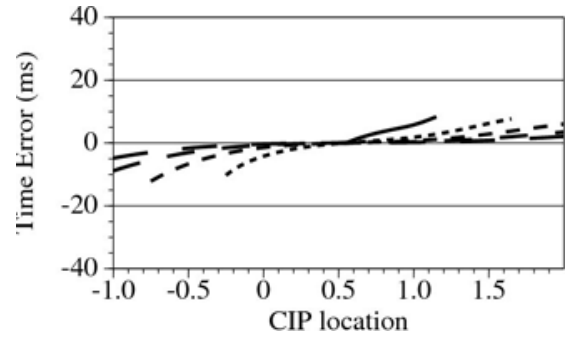

(c)

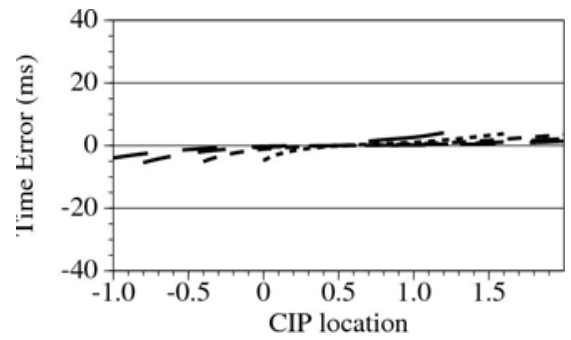

(e)

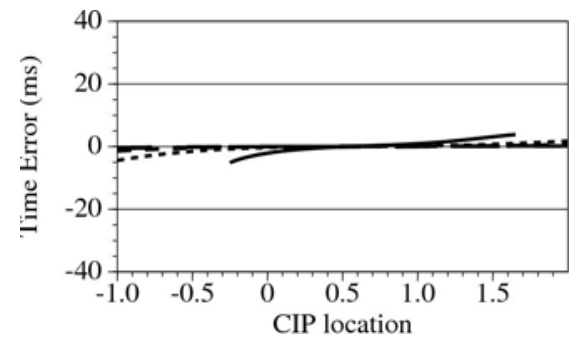

(g)

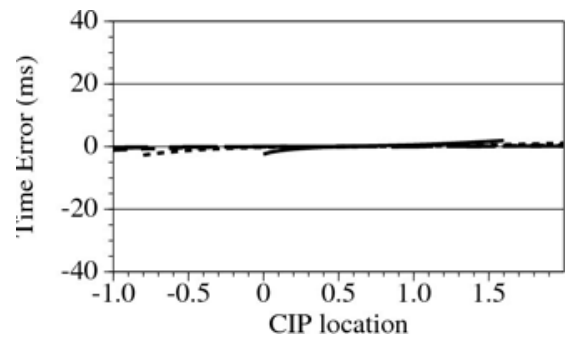

(b)

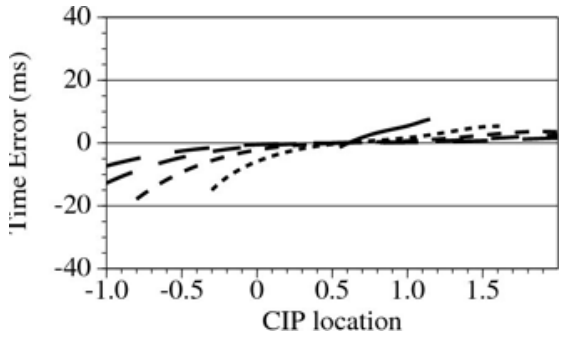

(d)

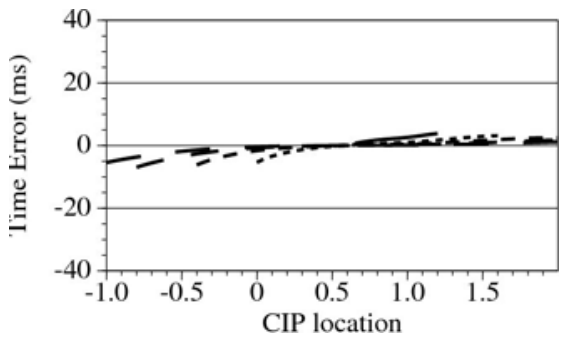

(f)

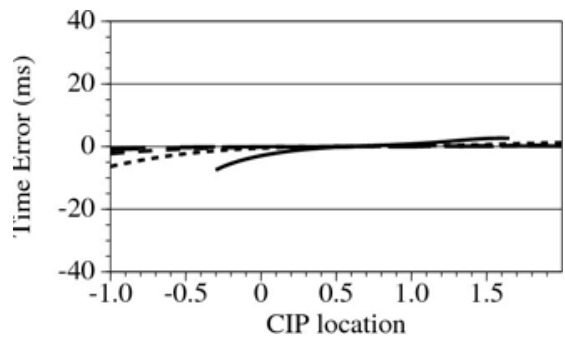

(h)

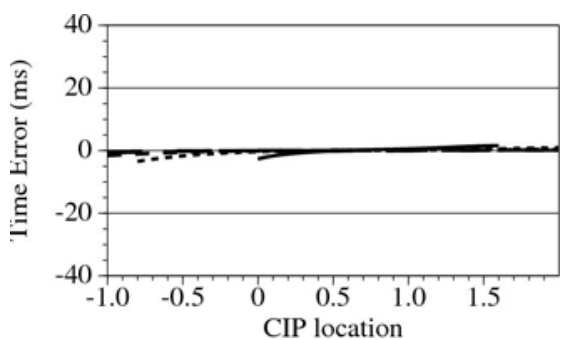

Figure 4. 
(a)

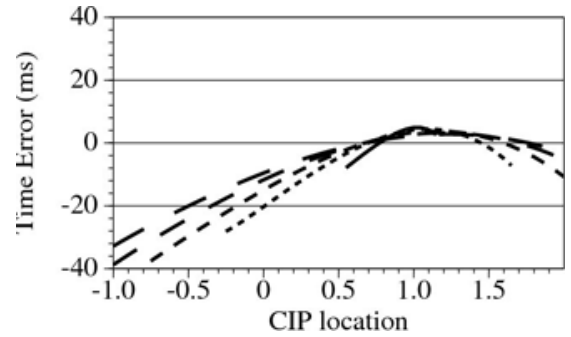

(c)

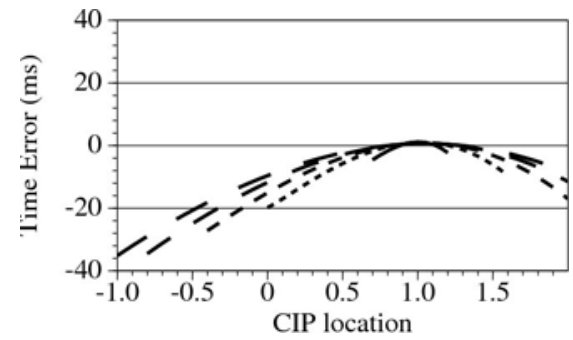

(e)

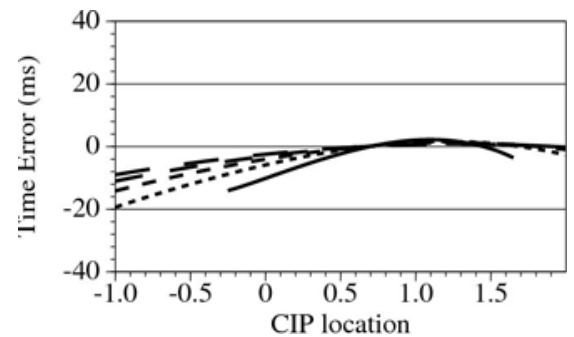

(g)

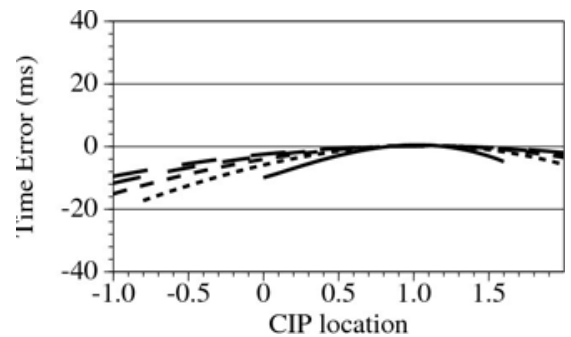

(b)

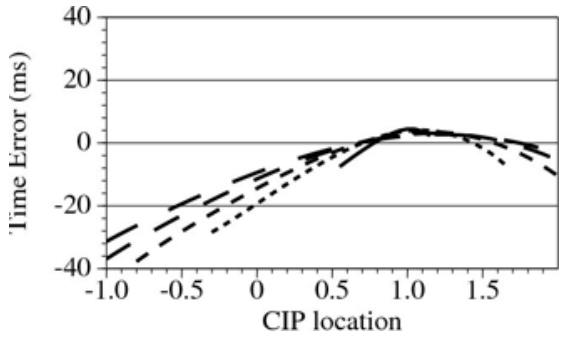

(d)

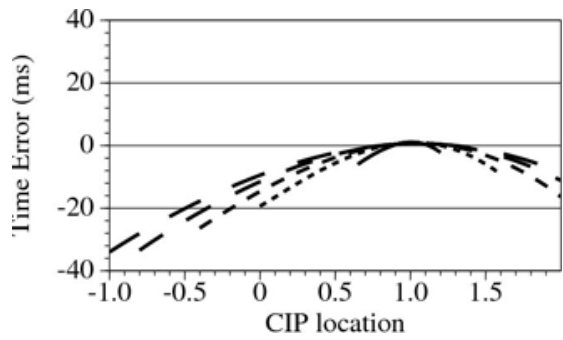

(f)

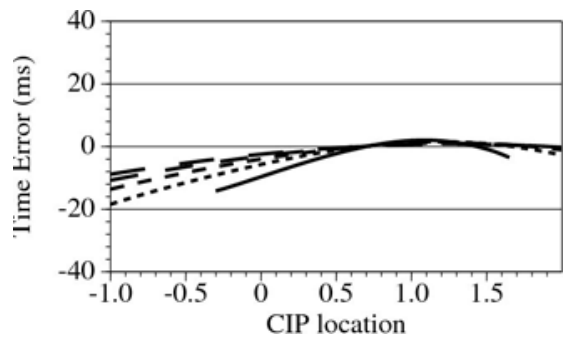

(h)

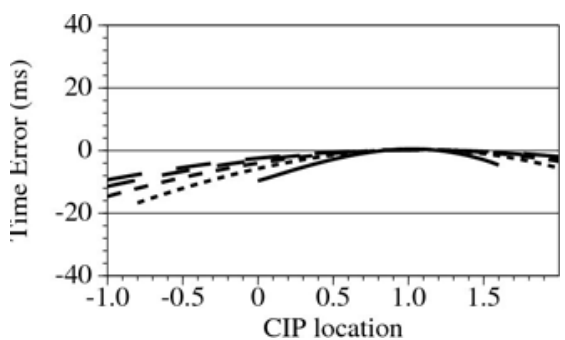

Figure 5. 
(a)

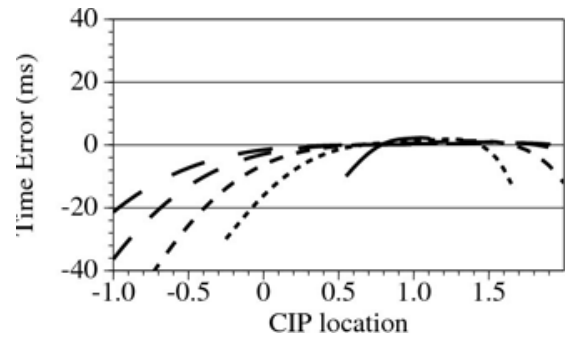

(c)

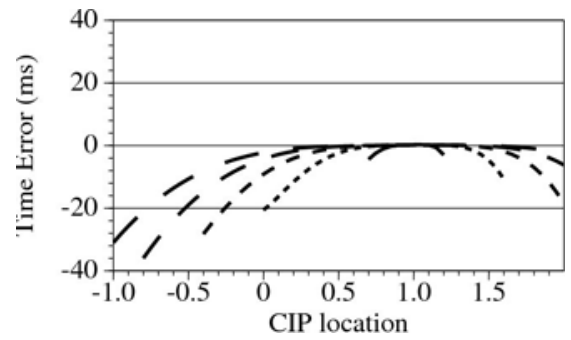

(e)

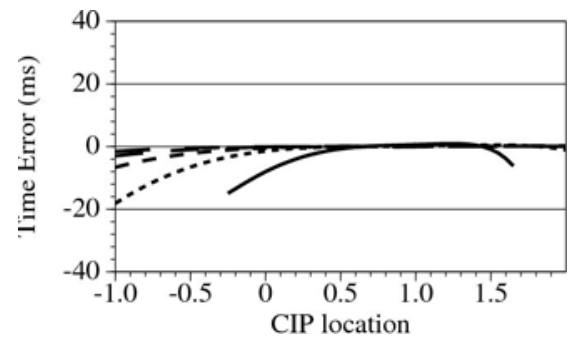

(g)

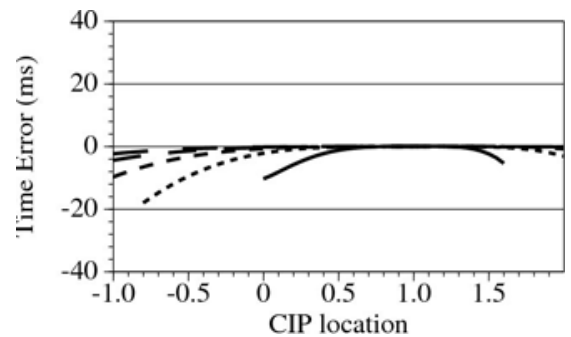

(b)

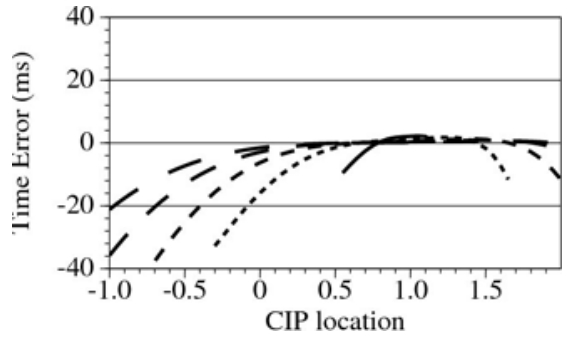

(d)

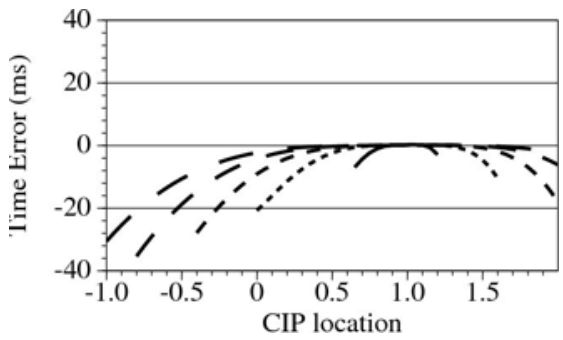

(f)

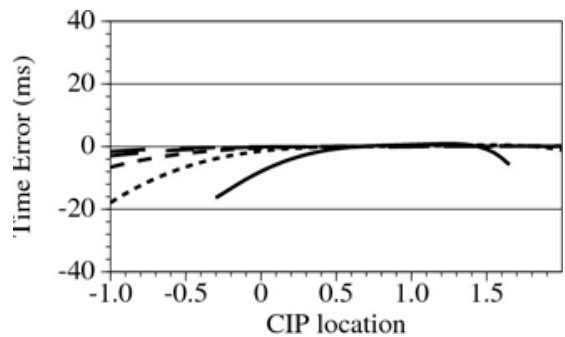

(h)

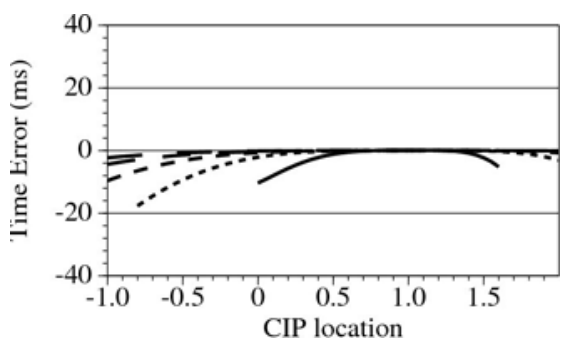

Figure 6. 


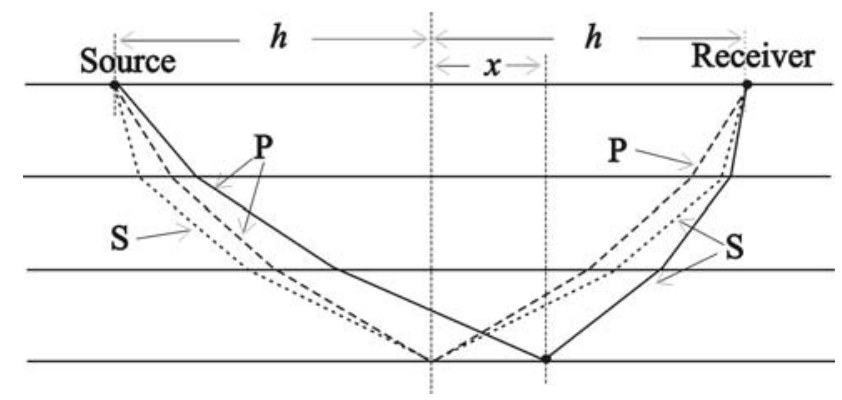

Figure 7. 


\section{Symbol list}

$V_{p 0 i}$ : Interval P-wave velocity.

$V_{s 0 i}$ : Interval S-wave velocity.

$\varepsilon_{i}$ : Interval Thomson's parameter

$\delta_{i}$ : Interval Thomson's parameter

$V_{p 2 i}$ : Interval short-spread moveout velocity for P-waves

$V_{s 2 i}$ : Interval short-spread moveout velocity for S-waves

$\sigma_{i}$ : Interval anisotropic parameter

$\eta_{i}$ : Interval anisotropic parameter for P-waves

$\zeta_{i}:$ Interval anisotropic parameter for S-waves

$t_{p 0 i}:$ Interval vertical travel-time of P-waves.

$t_{s 0 i}:$ Interval vertical travel-time of S-waves.

$V_{p 2}$ : RMS velocity of P-waves.

$V_{s 2}$ : RMS velocity of S-waves.

$\gamma_{0}:$ Vertical velocity ratio of $\mathrm{P}$ and S-waves.

$\gamma_{\text {eff }}$ : Effective velocity ratio of $\mathrm{P}$ and S-waves.

$V_{p s}:$ RMS velocity of PS-waves.

$\eta_{\text {eff }}:$ Effective anisotropic parameter for P-waves.

$\zeta_{\text {eff }}:$ Effective anisotropic parameter for S-waves.

$\chi_{\text {eff }}$ : Effective anisotropic parameter for PS-waves.

$t_{p}$ : Travel-time of P-waves from a source to a scatter-point.

$t_{s}$ : Travel-time of S-wave from a scatter-point to a receiver.

$t_{p s}$ : Two-way travel-time of PS-wave from a source to a receiver.

$t_{p 0}$ : Vertical travel-time of P-waves from a source to a scatter-point.

$t_{s 0}$ : Vertical travel-time of S-wave from a scatter-point to a receiver.

$t_{p s 0}$ : Two-way vertical travel-time of PS-wave from a source to a receiver.

$x_{p}$ : Horizontal distance between a source and a scatter-point.

$x_{S}$ : Horizontal distance between a scatter-point and a receiver.

$\Delta t_{p s}$ : Error in the travel-time of PS-waves. 
$E_{V_{p s}}:$ Error factor for $V_{p s}$.

$E_{V_{p s p}}$ : Part of $E_{V_{p s}}$ related to P-waves

$E_{V_{p s s}}:$ Part of $E_{V_{p s}}$ related to S-waves

$E_{\gamma_{0}}:$ Error factor for $\gamma_{0}$.

$E_{\gamma_{0 p}}$ : Part of $E_{\gamma_{0}}$ related to P-waves.

$E_{\gamma_{0 s}}:$ Part of $E_{\gamma_{0}}$ related to S-waves.

$E_{\text {reff }}$ : Error factor for $\gamma_{\text {eff }}$.

$E_{\gamma_{\text {efff }}}:$ Part of $E_{\gamma_{\text {eff }}}$ related to P-waves.

$E_{\text {reffs }}:$ Part of $E_{\gamma_{\text {eff }}}$ related to S-waves.

$E_{\chi}$ : Error factor for $\chi_{\text {eff }}$.

$E_{\chi p}$ : Part of $E_{\chi}$ related to P-waves.

$E_{\chi s}$ : Part of $E_{\chi}$ related to S-waves. 